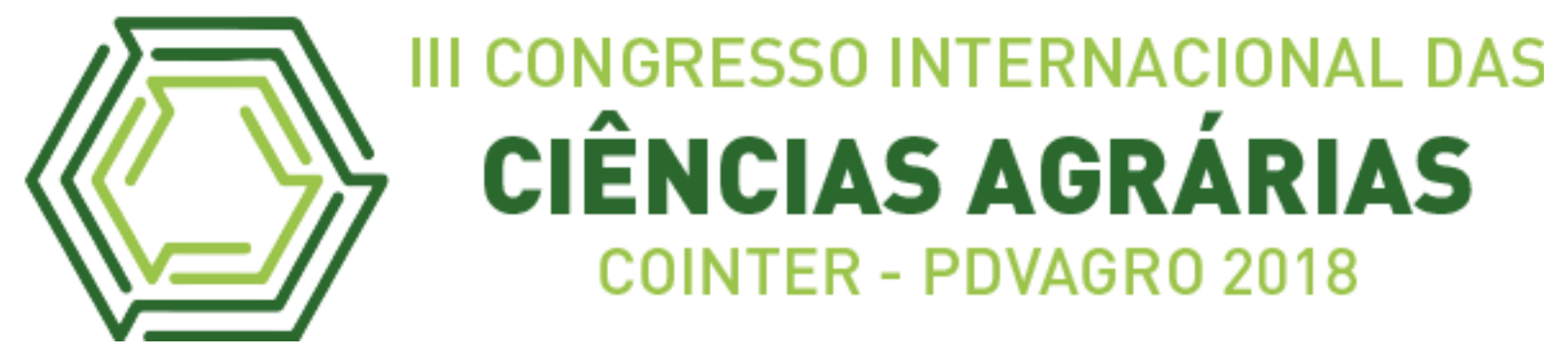

\title{
VERIFICAÇÃO DA CAPACIDADE DE ADSORÇÃO DE CÁDMIO E COBRE EM SOLOS DA BAIXADA MARANHENSE EM ÁREAS NATURAIS E ANTROPIZADAS.
}

\section{VERIFICATION OF ADDITION CAPACITY OF CÁDMIO AND COPPER IN SOILS OF BAJADA MARANHENSE IN NATURAL AND ANTROPIZED AREAS.}

\author{
Apresentação: Pôster
}

Naama Aguiar Moreira ${ }^{1}$; Jonas Juliermerson Silva Otaviano ${ }^{2}$; Ana Paula Brito de Sousa ${ }^{3}$; Rennan da Silva de Sousa Moreira ${ }^{4}$; Jorge Diniz de Oliveira ${ }^{5}$

DOI: https://doi.org/10.31692/2526-7701.IIICOINTERPDVAGRO.2018.00694

\section{Introdução}

Os metais potencialmente tóxicos estão presentes naturalmente nos solos e em rochas desde o seu processo de formação provenientes sobretudo de intemperismos químicos e biológicos. Entretanto, diversas áreas tem apresentado gradativamente maiores concentrações desses metais, somando-se a isso a transferência para a cadeia alimentar que atinge, portanto, organismos, plantas e animais presentes em determinado ambiente contaminado e ao homem. O aumento dos teores destes metais está associado à aplicação de corretivos e fertilizantes agrícolas, a produtos como lodo de esgoto, compostos de lixo urbano e resíduos de indústria ou mineração, e a utilização de água de irrigação poluída e/ou contaminada (COSTA, 2005; ALLEONI et al., 2005). Objetivou-se com este trabalho avaliar a adsorção competitiva de Cobre e Cádmio bem como verificar a contaminação em solos representativos da Baixada Maranhense usando a técnica de isotermas de adsorção.

\footnotetext{
${ }^{1}$ Engenharia Florestal; Universidade da Região Tocantina do Maranhão - UEMASUL Campus Imperatriz; naama.moreira@hotmail.com

${ }^{2}$ Engenharia Florestal; Universidade da Região Tocantina do Maranhão - UEMASUL Campus Imperatriz; jonas-eng.florestal@hotmail.com

${ }^{3}$ Engenharia Florestal; Universidade da Região Tocantina do Maranhão - UEMASUL Campus Imperatriz; paulabrito.1619@gmail.com

${ }^{4}$ Engenharia Florestal; Universidade da Região Tocantina do Maranhão - UEMASUL Campus Imperatriz; renanmoreira_sousa@hotmail.com

${ }^{5}$ Doutor em Química, Professor adjunto IV/ Centro de Ciências Exatas, Naturais e Tecnológicas - CCENT; Universidade Estadual da Região Tocantina do Maranhão - UEMASUL Campus Imperatriz; jzinid@hotmail.com
} 


\section{Fundamentação Teórica}

Os elementos cobre $(\mathrm{Cu})$ e cádmio $(\mathrm{Cd})$, normalmente encontram-se associados à poluição, à contaminação e toxidade. No entanto, o $\mathrm{Cu}$ é essencial aos seres vivos e por atender aos critérios de essencialidade para as plantas, é classificado como micronutriente. Apesar disto, este elemento, quando presente em altas concentrações no solo pode apresentar caráter tóxico. $\mathrm{O} \mathrm{Cd}$, por sua vez, é considerado um elemento potencialmente tóxico para homens, animais e plantas (CHAVES et al., 2009).

O cádmio pode ser adicionado aos solos por meio da aplicação de lodo de esgoto, adubação fosfatada ou qualquer forma de poluição, como, por exemplo, resíduos provenientes de indústrias e mineração. No solo, o Cd pode ser biodisponibilizado, acumulando-se nas plantas, ou lixiviado, poluindo as águas subterrâneas e comprometendo, assim, os suprimentos de água potável (PIERANGELI et al., 2005).

É relevante compreender, portanto, o nível de competitividade na biodisponibilidade destes metais no solo e sua capacidade de mobilidade e interação entre outros metais, seja de forma lixiviada ou absorvida pelas plantas e outros organismos

\section{Metodologia}

Foram empregadas amostras de solos provenientes do Banco de Dados do projeto Fauna de Califorídeos e Sarcofagídeos em três diferentes zonas fitogeográficas do estado do Maranhão. Foi estimado parâmetros físico-químicos tais como teor de matéria orgânica, carbono orgânico, teor de umidade, p.H, óxido de ferro, sendo que todas foram feitas em triplicata. Em seguida, foi realizado o estudo de capacidade de adsorção de cobre e cádmio em ambiente antropizado e ambiente natural em áreas da Baixada Maranhense utilizando isotermas de adsorção.

Método para os ensaios de adsorção

Foram preparadas soluções-padrão de concentrações crescentes de $\mathrm{CdCl}_{2}$ e $\mathrm{CuCl}_{2}$ e uma solução-padrão de $\mathrm{CaCl}_{2}$ 0,01 mol L ${ }^{-1}$. As soluções-padrão das espécies metálicas foram preparadas de modo que em uma razão solo: solução de 1:30 as concentrações iniciais das respectivas espécies metálicas na solução de equilíbrio serão $0,10,25,40,70,110,150$ e 200 $\mathrm{mg} \mathrm{L} \mathrm{L}^{-1}$. Foram pesadas alíquotas de $1,000 \mathrm{~g}$ de solo e peneiradas a $0,35 \mathrm{~mm}$ em erlenmeyers, em seguida foi adicionado $20 \mathrm{~mL}$ da solução de $\mathrm{CaCl}_{2} 0,01 \mathrm{~mol} \mathrm{~L}{ }^{-1}$. Todas as amostras foram submetidas agitação constante de $200 \mathrm{rpm}$ por 24 horas. Em seguida foi adicionado às 
amostras $10 \mathrm{~mL}$ das soluções-padrão do metal. As amostras foram submetidas a agitação constante de $200 \mathrm{rpm}$ por 24 horas posteriores. Decorrido o tempo de agitação o sobrenadante foi filtrado em membrana filtrante $0,45 \mathrm{~mm}$, o filtrado foi condicionando em um frasco de plástico de cor escura e guardado sob-refrigeração até o momento da determinação quantificação das espécies metálicas. A quantificação no metal na solução filtrada foi feita utilizando-se o Espectrômetro de Absorção Atômica por Atomização por Chama (FAAS), no comprimento de onda do metal específico.

\section{Resultados e Discussões}

É chamada de isoterma de adsorção a relação gráfica entre a concentração de íons adsorvidos pela fase sólida e concentração de cádmio e cobre na solução final ou de equilíbrio Ce.

O ajuste de resultados experimentais a modelos de adsorção fornece informações importantes sobre a capacidade de retenção e a força pela qual o adsorvido está preso ao solo. A característica de reter moléculas em uma determinada superfície e o grau com que elas se acentuam caracterizam o estudo de adsorção, esse fenômeno pode ocorrer em diversas interfaces do analito, tanto Gás-solido, solução-gás, solução $\alpha$-solução $\beta$ ou como no caso estudado solução-solido (QFL 0406 IQ-USP). A capacidade de adsorção varia de acordo ao material adsorvido, convém lembrar que a superfície de um solido é composta por uma grande número de sítios, e que cada sítio pode ser ocupado por uma molécula adsorvida logo adsorção só será completa se todos os sítios forem ocupados, formando uma monocamada, além disso, todos os experimentos de adsorção foram realizados em p.H 5.

Foi realizado o estudo de capacidade de adsorção de cobre e cádmio em ambiente antropizado e ambiente natural em áreas da Baixada Maranhense utilizando isotermas de adsorção. No estudo das isotermas (Figuras 1, 2 e 3) observou-se na maioria das curvas uma menor inclinação, sobretudo na parte inicial. De acordo com as figuras representadas, todas as quantidades de $\mathrm{Cu}$ (II) e $\mathrm{Cd}$ (II) adsorvidas aumentaram com o aumento das doses adicionadas.

Figura 1: Isoterma de adsorção de Cu (II) e Cd (II) no sistema competivo para o solo de Cajapió em ambiente
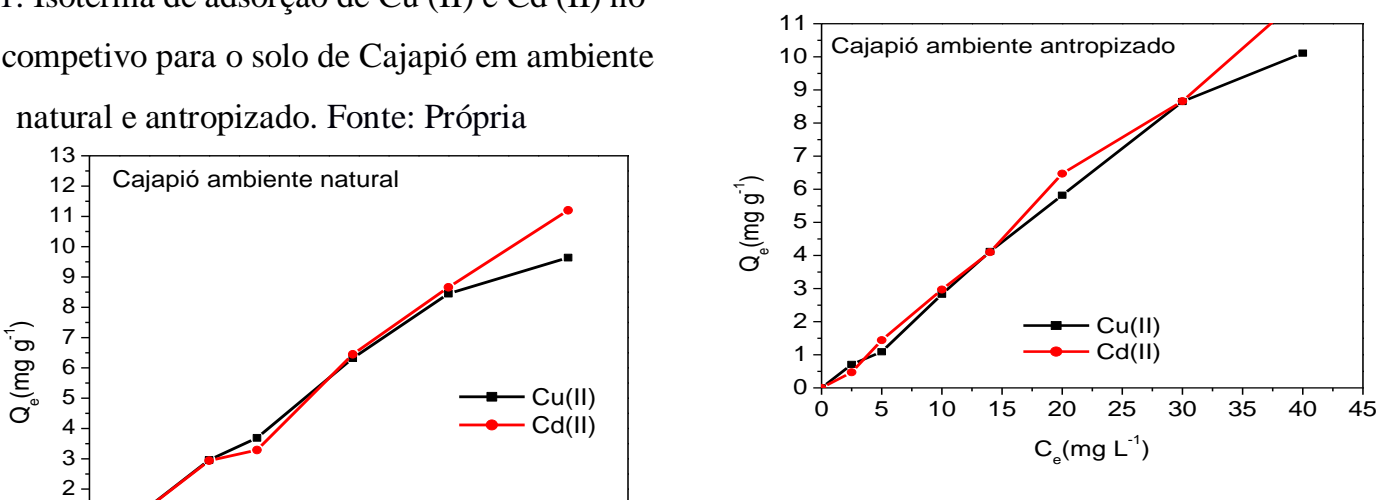
Figura 2. Isoterma de adsorção de Cu (II) e Cd (II) no sistema competivo para o solo de Cajari em ambiente natural e antropizado. Fonte: Própria
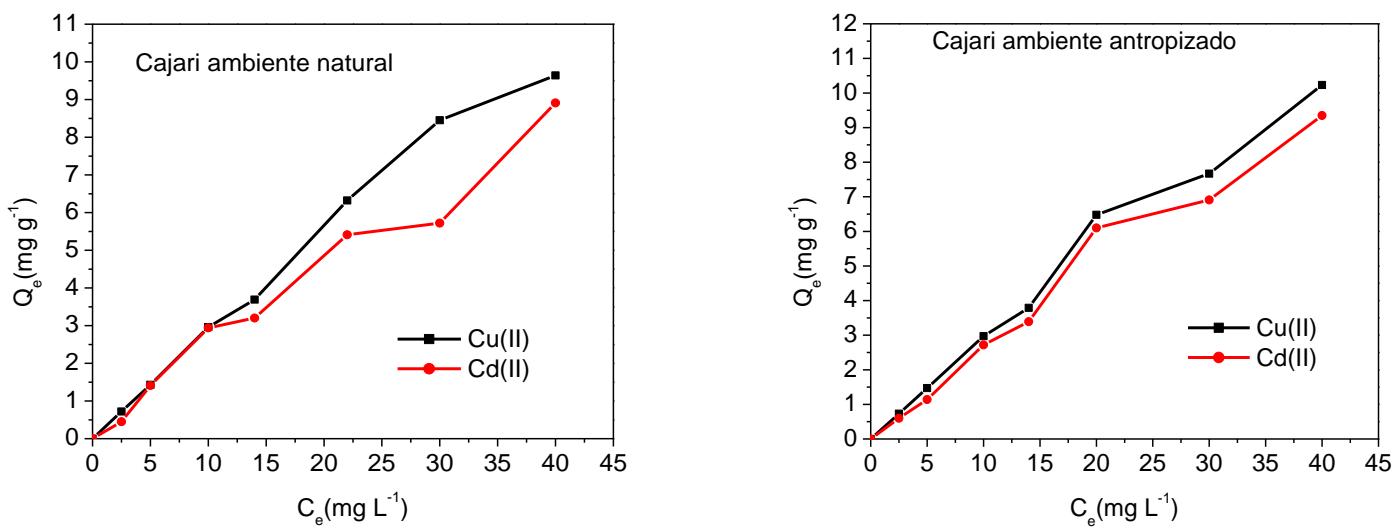

Figura 3. Isoterma de adsorção de Cu (II) e Cd (II) no sistema competivo para o solo de Viana em ambiente natural e antropizado. Fonte: Própria.
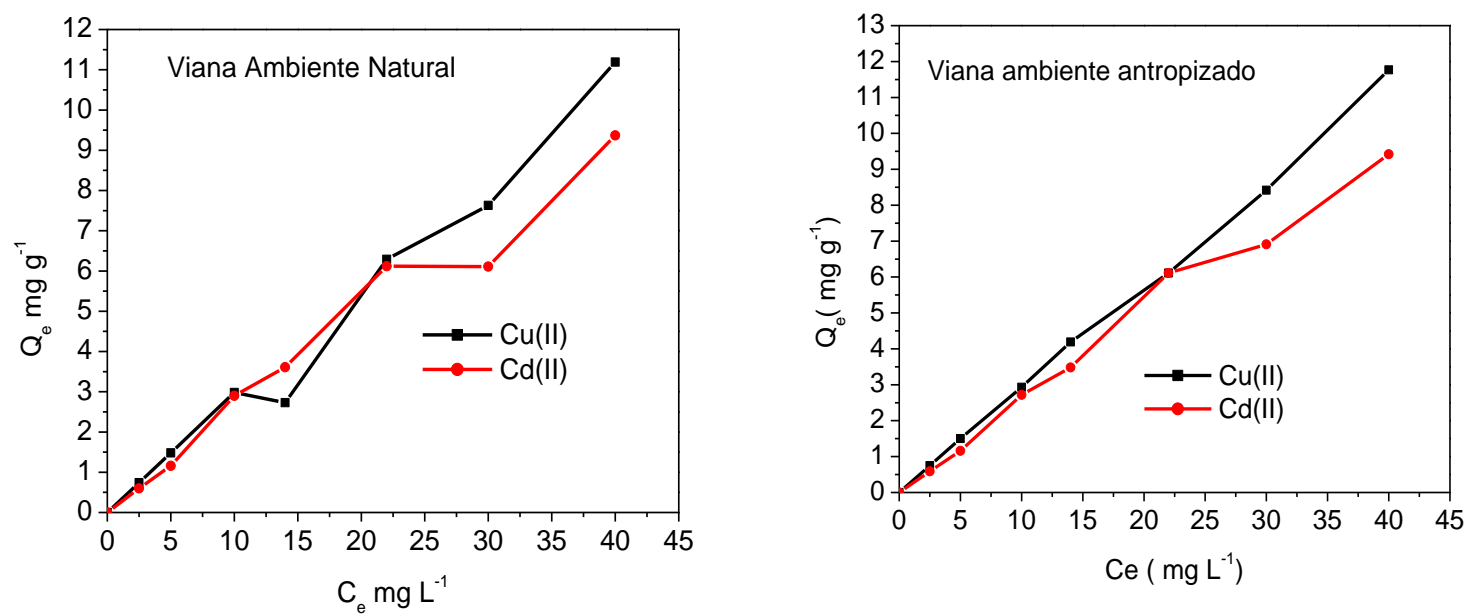

No processo competitivo, a composição iônica da solução exerce amplo efeito na adsorção de íons nas partículas do solo (Muraly \& Ailmore, 1983; Echeverría et al., 1998;). No entanto, o que causa esse efeito é a competitividade entre os íons de solução e aqueles adsorvidos na superfície do solo e diferentes sequências de afinidade devido a uma série de fatores inserindo o tipo de adsorbato e condições experimentais (Moreira, C. S. et al, 2004). A utilização das isotermas de adsorção de Langmuir e Freundlich nos solos de Cajapió, Cajari e Viana simularam de maneira satisfatória tanto para os íons $\mathrm{Cu}^{2+}$ quanto para os íons de $\mathrm{Cd}^{2+}$. $\mathrm{O} \mathrm{Cu}^{2+}$ foi adsorvido preferencialmente, ou seja, $\mathrm{Cu}^{2+}>\mathrm{Cd}^{2+}$ além disso, o Cobre interfere também na retenção do Cádmio. No sistema competitivo, a superioridade do Cobre foi maior 
que a do Cádmio. Portanto, esse comportamento entre os metais indicou que a adsorção simultânea é considerada para verificar a toxicidade tal como o potencial de lixiviação dos metais potencialmente tóxicos no solo.

Tabela 1. Comparação estatística da capacidade de adsorção de $\mathrm{Cu}$ e $\mathrm{Cd}$ do solo de Cajapió em área natural e antropizadas em diferentes dosagem. As médias seguidas pela mesma letra minúscula na coluna e maiúscula na

linha não diferem estatisticamente entre si. Foi aplicado o Teste de Tukey ao nível de 5\% de probabilidade. Fonte: Própria.

\begin{tabular}{|c|c|c|c|c|c|c|c|c|}
\hline \multirow{2}{*}{ Área } & \multicolumn{8}{|c|}{ Dosagem adicionada $\left(\mathrm{mg} \mathrm{L}^{-1}\right)$} \\
\cline { 2 - 9 } & & 2,5 & 5 & 10 & 14 & 22 & 30 & 40 \\
\hline \multirow{3}{*}{ Natural } & $\mathrm{Cu}$ & $0,72 \mathrm{aE}$ & $1,43 \mathrm{aE}$ & $2,95 \mathrm{aD}$ & $3,69 \mathrm{abD}$ & $6,32 \mathrm{aC}$ & $8,45 \mathrm{aB}$ & $10.11 \mathrm{bA}$ \\
\cline { 2 - 9 } & $\mathrm{Cd}$ & $0,49 \mathrm{aF}$ & $1,42 \mathrm{aE}$ & $2,94 \mathrm{aD}$ & $3,29 \mathrm{bD}$ & $6,45 \mathrm{aC}$ & $7,66 \mathrm{bB}$ & $11,20 \mathrm{aA}$ \\
\hline \multirow{3}{*}{ Antropizada } & $\mathrm{Cu}$ & $0,72 \mathrm{aF}$ & $1,09 \mathrm{aF}$ & $2,83 \mathrm{aE}$ & $4,12 \mathrm{aD}$ & $6,15 \mathrm{aC}$ & $8,65 \mathrm{aB}$ & $10,10 \mathrm{bA}$ \\
\cline { 2 - 9 } & $\mathrm{Cd}$ & $0,47 \mathrm{aF}$ & $1,44 \mathrm{aE}$ & $2,97 \mathrm{aCD}$ & $3,20 \mathrm{bC}$ & $5,29 \mathrm{bB}$ & $2,12 \mathrm{cDE}$ & $9,35 \mathrm{cA}$ \\
\hline
\end{tabular}

Tabela 2. Comparação estatística da capacidade de adsorção de $\mathrm{Cu}$ e $\mathrm{Cd}$ do solo de Cajari em área natural e antropizadas em diferentes dosagem. As médias seguidas pela mesma letra minúscula na coluna e maiúscula na

linha não diferem estatisticamente entre si. Foi aplicado o Teste de Tukey ao nível de 5\% de probabilidade. Fonte: Própria.

\begin{tabular}{|c|c|c|c|c|c|c|c|c|}
\hline \multirow{3}{*}{ Área } & \multicolumn{8}{|c|}{ Dosagem adicionada $\left(\mathrm{mg} \mathrm{L}^{-1}\right)$} \\
\cline { 2 - 9 } & & 2,5 & 5 & 10 & 14 & 22 & 30 & 40 \\
\hline \multirow{3}{*}{ Natural } & $\mathrm{Cu}$ & $0,74 \mathrm{aG}$ & $1,47 \mathrm{aF}$ & $2,95 \mathrm{aE}$ & $4,11 \mathrm{aD}$ & $6,46 \mathrm{aC}$ & $7,55 \mathrm{bB}$ & $10,25 \mathrm{aA}$ \\
\cline { 2 - 9 } & $\mathrm{Cd}$ & $0,45 \mathrm{eG}$ & $1,41 \mathrm{bF}$ & $2,94 \mathrm{aE}$ & $3,20 \mathrm{dD}$ & $5,41 \mathrm{cC}$ & $5,72 \mathrm{~dB}$ & $8,91 \mathrm{cA}$ \\
\hline \multirow{2}{*}{ Antropizada } & $\mathrm{Cu}$ & $0,73 \mathrm{aG}$ & $1,47 \mathrm{aF}$ & $2,97 \mathrm{aE}$ & $3,79 \mathrm{bD}$ & $6,48 \mathrm{aC}$ & $7,76 \mathrm{aB}$ & $10,23 \mathrm{aA}$ \\
\cline { 2 - 9 } & $\mathrm{Cd}$ & $0,60 \mathrm{bG}$ & $1,14 \mathrm{cF}$ & $2,72 \mathrm{bE}$ & $3,39 \mathrm{cD}$ & $6,10 \mathrm{bC}$ & $6,91 \mathrm{cB}$ & $9,35 \mathrm{bA}$ \\
\hline
\end{tabular}

Tabela 3. Comparação estatística da capacidade de adsorção (Qe) de Cu e Cd do solo de Viana em área natural e antropizadas em diferentes dosagem. As médias seguidas pela mesma letra minúscula na coluna e maiúscula na

linha não diferem estatisticamente entre si. Foi aplicado o Teste de Tukey ao nível de 5\% de probabilidade. Fonte: Própria.

\begin{tabular}{|c|l|c|c|c|c|c|c|l|}
\hline \multirow{2}{*}{ Área } & \multicolumn{8}{|c|}{ Dosagem adicionada $\left(\mathrm{mg} \mathrm{L}^{-1}\right)$} \\
\cline { 2 - 10 } & & 2,5 & 5 & 10 & 14 & 22 & 30 & \multicolumn{1}{c|}{40} \\
\hline \multirow{2}{*}{ Natural } & $\mathrm{Cu}$ & $0,74 \mathrm{aG}$ & $1,48 \mathrm{Af}$ & $2,98 \mathrm{aD}$ & $2,73 \mathrm{dE}$ & $6,29 \mathrm{aC}$ & $7.63 \mathrm{bB}$ & $11,19 \mathrm{bA}$ \\
& $\mathrm{Cd}$ & $0,60 \mathrm{bF}$ & $1,16 \mathrm{bE}$ & $2,90 \mathrm{cD}$ & $3,61 \mathrm{bC}$ & $6,12 \mathrm{bB}$ & $6,11 \mathrm{Db}$ & $9,17 \mathrm{dA}$ \\
\hline \multirow{2}{*}{ Antropizada } & $\mathrm{Cu}$ & $0,75 \mathrm{aG}$ & $1,50 \mathrm{Af}$ & $2,93 \mathrm{bE}$ & $4,19 \mathrm{aD}$ & $6,11 \mathrm{bC}$ & $8,42 \mathrm{Ab}$ & $11,77 \mathrm{aA}$ \\
& $\mathrm{Cd}$ & $0,59 \mathrm{bG}$ & $1,16 \mathrm{Bf}$ & $2,72 \mathrm{dE}$ & $3,48 \mathrm{cD}$ & $6,11 \mathrm{bC}$ & $6,91 \mathrm{Cb}$ & $9,42 \mathrm{cA}$ \\
\hline
\end{tabular}

De acordo com a análise estatística das tabelas 1, 2 e 3 em áreas naturais e antrópicas dos municípios de Cajapió, Cajari e Viana indica que o $\mathrm{Cu}$ (II) em grande parte apresentou 
maior capacidade de adsorção comparado aos valores de Cd (II), especialmente nas dosagens 14,30 e 40. Não houve diferenças significativas entre os metais na pequenas dosagens. Na tabela 3, o doseamento 22 e 10 de ambiente natural e antropizado, respectivamente, a capacidade de adsorção de $\mathrm{Cd}$ (II) foi ligeiramente maior que $\mathrm{Cu}$ (II).

\section{Conclusões}

Todos os parâmetros físico-químicos do solo influenciaram na capacidade de adsorção. A utilização das isotermas de adsorção de Langmuir e Freundlich nos solos de Cajapió, Cajari e Viana simularam de maneira satisfatória tanto para os íons $\mathrm{Cu}^{2+}$ quanto para os íons de $\mathrm{Cd}^{2+}$. $\mathrm{O} \mathrm{Cu}^{2+}$ foi adsorvido preferencialmente, ou seja, $\mathrm{Cu}^{2+}>\mathrm{Cd}^{2+}$. O comportamento entre os metais potencialmente tóxicos indicou que a adsorção simultânea é relevante para verificar a toxicidade tal como o potencial de lixiviação dos metais pesados no solo. No sistema competitivo, a superioridade do Cobre foi maior que a do Cádmio.

\section{Referências}

ALLEONI, L. R. F.; IGLESIAS, C. S. M.; MELLO, S. C.; CAMARGO, O. A.; ARIAS, M. et al. Adsorption and desorption of copper and zinc in the surface layer of acid soils. Colloid and Interface Science v. 288, n. 01, p. 21-29, 2005.

COSTA, C.N. Biodisponibilidade de metais pesados em solos do Rio Grande do Sul. 2005. 110f. Tese (Doutorado) - Programa de Pós- Graduação em Ciência do Solo, Faculdade de Agronomia, Universidade Federal do Rio Grande do Sul, Porto Alegre, 2005.

CHAVES, L. H. G,; SOUZA, R.S.; CHAVES, I, B.; TITO, G. A. Adsorção de metis pesados em luvissolos e cambissolos do estado da Paraíba. Engenharia Ambiental, v. 6, n. 2, p. 150$162,2009$.

PIERANGELI, M. P. P.; GUILHERME GUIMARÃES, L. R.; CURI, M.; SILVA, M. L N.; LIMA, J. M.; COSTA, E. T. S. Efeito do ph na adsorção e dessorção de cádmio em latossolos brasileiros. Revista brasileira de Ciências do Solo,. n. 29, p.:523-532, 2005. 